\title{
PLASTIC DEFORMATION IN LASER-INDUCED SHOCK COMPRESSION OF MONOCRYSTALLINE COPPER
}

\author{
M. A. Meyers $^{1}$, F. Gregori ${ }^{2}$, B. K. Kad ${ }^{1}$, M. S. Schneider ${ }^{1}$, D. H. Kalantar ${ }^{3}$, B. A. \\ Remington $^{3}$, J. S. Wark ${ }^{4}$, and T. Boehly ${ }^{5}$, G. Ravichandran ${ }^{6}$ \\ ${ }^{1}$ University of California, San Diego, La Jolla, CA, 92092, ${ }^{2}$ University of Paris, 13, Paris, France \\ ${ }^{3}$ Lawrence Livermore National Laboratory, Livermore, CA, 94551, ${ }^{4}$ Oxford University, Oxford, UK \\ ${ }^{5}$ University of Rochester, Rochester, NY, 14627, ${ }^{6}$ California Institute of Technology, Pasadena, CA 91125
}

\begin{abstract}
Copper monocrystals were subjected to shock compression at pressures of 10-60 GPa by a short (3 ns initial) duration laser pulse. Transmission electron microscopy revealed features consistent with previous observations of shock-compressed copper, albeit at pulse durations in the $\mu$ s regime. The results suggest that the defect structure is generated at the shock front. A mechanism for dislocation generation is presented, providing a realistic prediction of dislocation density as a function of pressure. The threshold stress for deformation twinning in shock compression is calculated from the constitutive equations for slip, twinning, and the Swegle-Grady relationship.
\end{abstract}

\section{INTRODUCTION}

Simultaneous shock compression and X-ray diffraction experiments were introduced by Johnson et al. [1] and continued by Zaretsky et al. [2] and Wark et al. [3]. These shock compression experiments on silicon monocrystals were coupled with X-ray diffraction that measured the compression. The OMEGA ICF (inertial confinement fusion) facility at the University of Rochester was used in a series of experiments on copper single crystals. Initial pulse durations were on the order of 2-5 ns, one order of magnitude lower than earlier shock laser experiments (20-100 ns) and two orders of magnitude lower than plate impact experiments $(0.1-2 \mu \mathrm{s})$. Thus, these experiments explore a new regime of shock compression.

\section{EXPERIMENTAL TECHNIQUES}

Single crystals with a [100] orientation were obtained from Goodfellow in the form of disks with $2 \mathrm{~mm}$ diameter and $1 \mathrm{~mm}$ thickness. They were mounted into recovery chambers shown in Figure 1.
Foam was used as a sample deceleration medium Shock compression was achieved by the vaporization of the single crystal surface layer due to a short duration high intensity laser beam, as shown in Figure 1. The results of three experiments are reported here: (a) energy $=40 \mathrm{~J}$ directly onto $\mathrm{Cu}$ (b) energy $=205 \mathrm{~J}$ directly onto $\mathrm{Cu}$; (c) energy = $320 \mathrm{~J}$ onto $20 \mu \mathrm{m} \mathrm{CH}$ ablator on $\mathrm{Cu}$. The respective initial pressures are approximately 12,40 , and 55 $60 \mathrm{GPa}$. Although these values decay throughout the specimens, they are used in the following discussions.

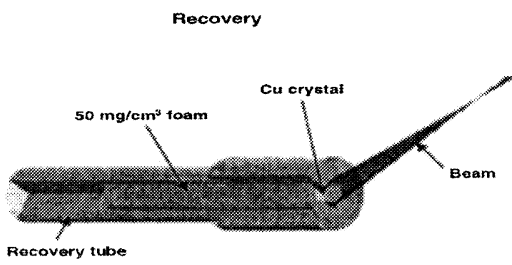

FIGURE 1. Sample and recovery chamber 
The shocked specimens were sliced into 3 discs using a slow-speed diamond saw and were ground mechanically to a thickness of $100 \mu \mathrm{m}$. Thin foils for TEM examination were prepared by using a twin-jet Fishione polisher. TEM investigations were conducted in a Philips CM-30 microscope operating at $300 \mathrm{kV}$.

\section{EXPERIMENTAL RESULTS AND DISCUSSION}

\section{Laser Energy of 40J (Pressure of 12 GPa)}

This low energy shock creates a cellular organization of a low density of $1 / 2<110>$ dislocations. The cells are fairly homogeneous, Figure 2, with average dimensions between 0.3 $0.6 \mu \mathrm{m}$. In thicker areas, away from the foil edge, the microstructure consists of dense dislocation cells. The results obtained in general confirm previous observations, albeit at a pulse duration that is lower by a factor of $10-100$, than that applied by Murr [5]. The predicted cell size from Murr's plot, at a pressure of $12 \mathrm{GPa}$, is $0.4 \mu \mathrm{m}$. The observed cell size is also consistent with Gray's [5] measurements: $0.5 \mu \mathrm{m} / 10 \mathrm{GPa}$.

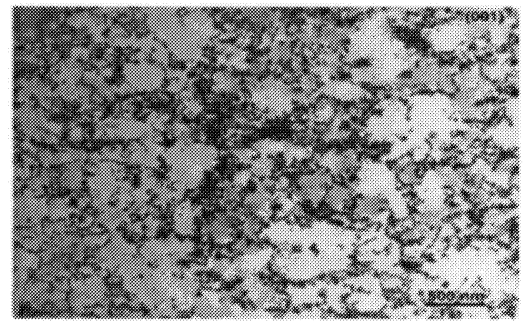

FIGURE 2. Dislocation substructure for the $\mathrm{Cu}$ specimens shocked at $40 \mathrm{~J}$ energy. $\mathrm{B}=[001]$

\section{Laser Energy of 205J (Pressure of 40 GPa)}

This intermediate energy input creates dense dislocation tangles, stacking faults and micro-twins. There are no readily discernible dislocation cells. Furthermore, the observed deformation substructure was nearly uniform around the thin foil perforation. These traces are characteristic of stacking-fault bundles and twins and are analogous to previous observations by Murr [6], especially,
Figs. 20, 21, and 23 of [6]. These features are significantly different than the ones at the lower energy. Traces of planar features are seen when the beam direction is $<101>$ as shown by the arrows of Figure 3.

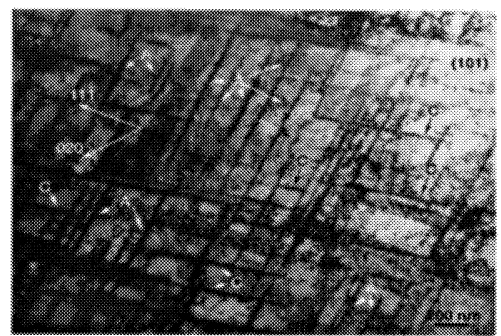

FIGURE 3. Stacking fault variant $\mathrm{A}$ imaged in the near edge orientation. Energy Input $=205$ Joules; $g=020 ; B=[001]$

\section{Laser Energy of 320J (Pressure of 60 GPa)}

The deformation microstructure consists of a high density of micro-twins, Figure 4, and laths, Figure 5. The deformation is not uniform around the perforation, with the micro-twins situated closer to the center of the sample and the laths away from the center.

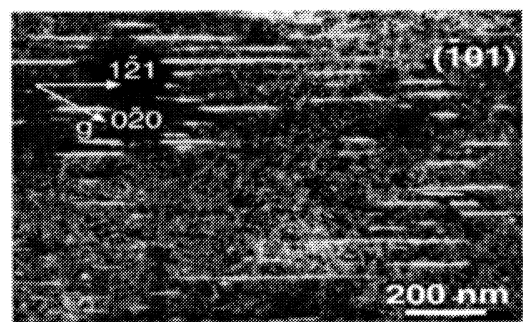

FIGURE 4. Micro-twins with a (111) habit plane elongated along $[1-21]$. Energy Input $=320$ Joules, $g=0-20, B=[101]$

Two sets of micro-twins are observed in the thin foils. We will focus our description only on one set. When imaged in the edge orientation at B close to [101], Figure 4, the micro-twins have the (111) habit plane and are elongated along [1-21] with an average $125 \mathrm{~nm}$. This set of micro-twins exhibit a wide range of lengths, from as small as $70 \mathrm{~nm}$ and as large as $1000 \mathrm{~nm}$. In contrast, the second set of micro-twins (not shown) is of a nearly uniform length of $70 \mathrm{~nm}$.

Unlike the micro-twins, the laths are elongated close to $\langle 220\rangle$. The lath interface plane is $\|[001]$ 
and therefore uniquely different from micro-twins. The laths also have been seen by Murr [5,6] and are due to thermal recovery at the shock compression. The residual temperature at the energy level is 550 $\mathrm{K}$.

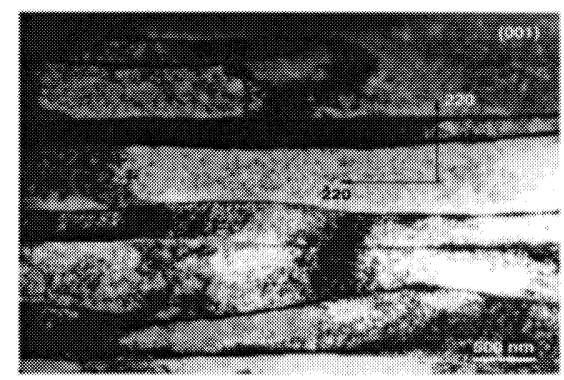

FIGURE 5. View of wavy lath interfaces imaged at $\mathrm{B}=[001]$ with the interface in the near edge orientation.

\section{ANALYSIS}

The observations made on recovered shock compressed copper specimens can be compared with analytical calculations that use physically based mechanisms. Two aspects in particular can be predicted: (a) the dislocation density as a function of peak shock amplitude and (b) the threshold stress for twinning.

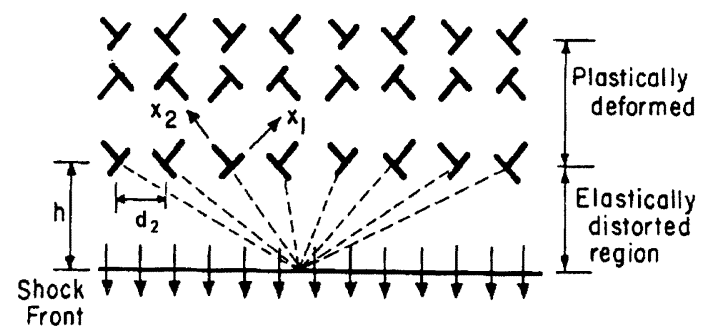

FIGURE 6. Stress due to dislocations on a point at shock front.

\section{(a) Dislocation Density}

The specific volume can be expressed as a function of pressure, $\mathrm{P}$, through one of the equations obtained directly from the RankineHugoniot equations and the equation of state [7].

$\frac{V}{V_{0}}=1-\frac{1}{2 P V S^{2}}\left[2 P V_{0}+C_{0} \pm \sqrt{\left(2 P V_{0}+C_{0}^{2}\right)^{2}-4 P^{2} V_{0}^{2} S^{2}}\right]$

$\mathrm{C}_{\mathrm{o}}$ and $\mathrm{S}$ are the material specific equation of state parameters. The spacing between the adjacent layers of dislocations in the homogeneous dislocation generation mechanism [8] is related to the dislocation density, $\rho$, by (Figure 6 shows the parameters $d_{2}$ and $h$ ):

$\rho=\left(\frac{d_{2} h}{2}\right)^{-1}$

Where $h=h_{1}$ for stationary dislocations and $h=h_{2}$ for dislocations traveling at the shear wave velocity $V_{\text {sf. }}$ The value of $h_{1}$ and $h_{2}$ can be estimated [10].

$h_{1}=\frac{1-v}{b \pi^{2}} d_{2}^{2}$

$h_{2}=h_{1} \frac{C_{0}}{V_{d p}}=2\left(\frac{1-v}{V_{s f}}\right)\left(\frac{V_{0}}{V}\right)^{1 / 2}\left(\frac{\lambda+2 \mu}{2 \mu}\right)^{1 / 2} d_{2}^{2}$

$\mathrm{V}_{\mathrm{d} p}$ is dislocation velocity component along the shock propagation direction, and $\mu$ and $\lambda$ are Lamé constants. Substitution of Eqn. (3) and (4) into (2) and (1), yield expressions for the pressure as a function of the dislocation density.

$$
P=\frac{C_{0}^{2}\left\{1-\left\{1-\sqrt{2}\left[\frac{b^{2}(1-v) \rho}{2 \pi^{2}}\right]^{1 / 3}\right\}^{3}\right\}}{V_{0}\left\{1-S\left\{1-\sqrt{2}\left[\frac{b^{2}(1-v) \rho}{2 \pi^{2}}\right]^{1 / 3}\right\}^{3}\right\}^{2}}
$$

One should be careful in interpreting these results, since recovery processes occur at pressures much lower than the maximum in the plot, and these high dislocation densities are not reached. The predicted dislocation densities (for the two cases $h_{1}$ and $h_{2}$ ) are plotted in Figure 7 and compared with observations by Murr [5,6].

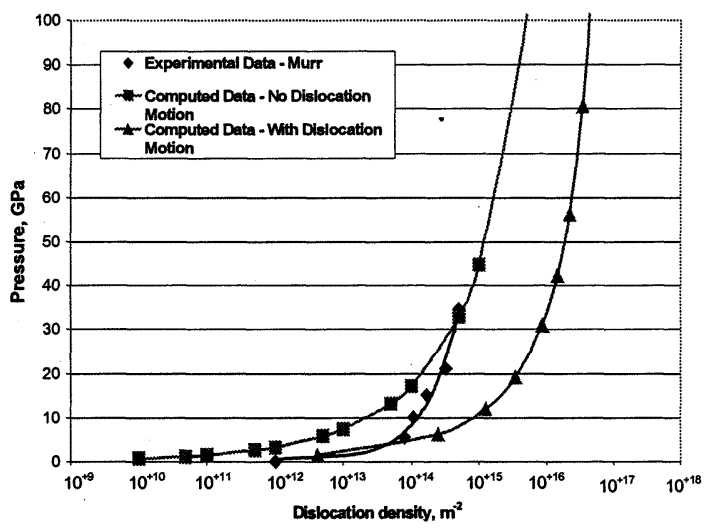

FIGURE 7. Experimental (from Murr [5]) and computed dislocation densities as a function of pressure for copper. 


\section{(b) Prediction of Threshold Amplitude for Twinning}

The procedure presented herein $[9,10]$ can be used to predict the critical pressure for twinning in shock compression experiments. It has been established by Murr [11] that this pressure is a function of stacking-fault energy, for FCC metals. Slip and twinning are considered as competing mechanisms; whereas plastic deformation by slip $\left(\sigma_{s}\right)$ has a strain rate and temperature dependence well described by the theory of thermally-activated obstacles, it is assumed that the strain rate and temperature dependence for twinning, $(\sigma T)$ are much lower. We first set:

$$
\sigma_{T}=\sigma_{s}
$$

The application of this criterion to the shock front necessitates the knowledge of the strain rate. The strain rate at the shock front has been established by Swegle and Grady [12] to be:

$$
P=k_{S G} \dot{\varepsilon}^{1 / 4}
$$

The constitutive response of the copper monocrystal is represented by the modified mechanical threshold stress (MTS) expression below; the parameters are taken from Follansbee and Gray [13]. The MTS model and parameters are defined by Follansbee and Kocks [14]. A modified MTS equation is used, with values of $p=1 / 2$ and $q=$ $3 / 2$, respectively [15]. The value of $g_{0}$ is 0.8 [16].

$$
\sigma=\sigma_{0} f(\varepsilon)\left[1-\left(\frac{k T}{G b^{3} g_{0}} \ln \left(\frac{\dot{\varepsilon}_{0}}{\dot{\varepsilon}}\right)\right)^{2 / 3}\right]^{2}+\mathrm{kd}^{-1 / 2}
$$

We apply Eqn. (6) to Eqn. (8), assuming a constant $\sigma_{\mathrm{T}}$. We find $\dot{\varepsilon}$ from Eqn. 8, which is inserted into Eqn (7). This provides a first estimate of the pressure, $\mathrm{P}$, where $f(\varepsilon)$ is an experimentally obtained stress-strain relationship (polynomial expression). This pressure is then used to calculate the shock strain and temperature through the Rankine-Hugoniot relations. This procedure is iterative. Figure 8 shows the application of this method to copper. The plot shows how the initial temperature and grain size affect the threshold shock pressure. The calculated threshold pressure for a monocrystal (10 $\mathrm{mm}$ grain size) shocked from an initial temperature of $300 \mathrm{~K}$ is $17 \mathrm{GPa}$. This compares favorably with experimental results by $\mathrm{De}$ Angelis and Cohen [4]: $14 \mathrm{GPa}$.

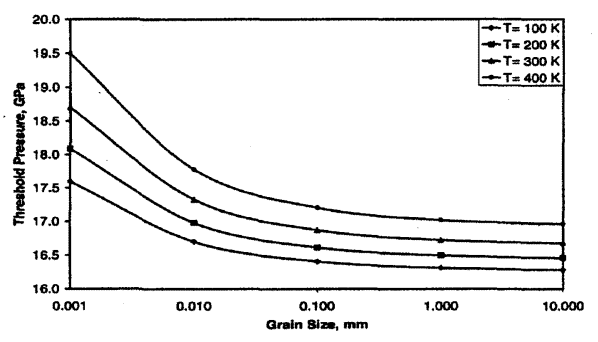

FIGURE 8. Predicted threshold stress for the initiation of twinning as a function of initial temperature and grain size

Research supported by the Department of Energy Grants DEFG0398DP00212 and DEFG0300SF2202.

\section{REFERENCES}

1. Johnson, Q., Mitchell, A., Keeler, R.N., and Evans, L., Phys. Rev. Lett., 25, 109 (1970).

2. Zaretsky,E., J. Appl. Phys., 78, 1.E (1995).

3. Wark, J.S.,et al. Phys. Rev. B, 40, 5705 (1989).

4. R.J. De Angelis, and J. B. Cohen, J. of Metals, 15, 681, (1963).

5. L.E. Murr, in Shock Waves and High-Strain-Rate Phenomena in Metals, eds. M.A. Meyers and L.E. Murr, Plenum, NY 1981, pp.607.

6. Murr, L. E., Scripta Met., 12, 201 (1978).

7. M. A. Meyers, Dynamic Behavior of Materials, J. Wiley, NY, 1994, pp. 149.

8. Meyers, M. A., Scripta Met., 13, 21 (1979)

9. L. E. Murr, M. A. Meyers, C. -S. Niou, Y. J. Chen, S. Pappu, and C. Kennedy, Acta Mater., 45, 157-75 (1997).

10. Meyers, M. A., et al., Matls. Sci. and Eng., in press (2001).

11. Murr, L. E., in Shock Waves in Condensed Matter, Eds. Schmidt, S. C. and Holmes, N. C., Elsevier, Amsterdam, (1988), pp. 315.

12. W. Swegle and D. E. Grady, J. Appl. Phys., 58941 (1983)

13. P. S. Follansbee and G. T. Gray III, Matls. Sci. and Eng. 138, 23 (1991).

14. P. S. Follansbee and U. F. Kocks, Acta Met., 36, 81 (1988).

15. P. S. Follansbee, in Metallurgical Applications of Shock-Wave and High-Strain Rate Phenomena ed, L. E. Murr, K. P. Staudhammer, and M. A. Meyers, M. Dekker. 1986, pp.451

16. Gray III, G.T., in "Shock-Wave and High-Strain-Rate Phenomena in Materials", eds. M.A. Meyers, L.E. Murr, and K.P. Staudhammer, M. Dekker, NY, 1992, pp. 899. 